\title{
Salvage carbon dioxide transoral laser microsurgery for laryngeal cancer after (chemo)radiotherapy: a European Laryngological Society consensus statement
}

\author{
Cesare Piazza, ${ }^{1,2}$ Alberto Paderno ${ }^{1,2}(\mathbb{D})$ Elisabeth V. Sjogren ${ }^{3}$. Patrick J. Bradley ${ }^{4}$ Hans E. Eckel . Antti Mäkitie $^{6}$. \\ Nayla Matar ${ }^{7} \cdot$ Vinidh Paleri $^{8} \cdot$ Giorgio Peretti ${ }^{9} \cdot$ Roberto Puxeddu $^{10} \cdot$ Miquel Quer $^{11} \cdot$ Marc Remacle $^{12}$. \\ Vincent Vander Poorten ${ }^{13,14} \cdot$ Isabel Vilaseca ${ }^{15} \cdot$ Ricard Simo $^{16}$
}

Received: 16 April 2021 / Accepted: 23 June 2021 / Published online: 5 July 2021

(c) The Author(s) 2021

\begin{abstract}
Purpose To provide expert opinion and consensus on salvage carbon dioxide transoral laser microsurgery $\left(\mathrm{CO}_{2} \mathrm{TOLMS}\right)$ for recurrent laryngeal squamous cell carcinoma (LSCC) after (chemo)radiotherapy [(C)RT].

Methods Expert members of the European Laryngological Society (ELS) Cancer and Dysplasia Committee were selected to create a dedicated panel on salvage $\mathrm{CO}_{2}$ TOLMS for LSCC. A series of statements regarding the critical aspects of decisionmaking were drafted, circulated, and modified or excluded in accordance with the Delphi process.

Results The expert panel reached full consensus on 19 statements through a total of three sequential evaluation rounds. These statements were focused on different aspects of salvage $\mathrm{CO}_{2}$ TOLMS, with particular attention on preoperative diagnostic work-up, treatment indications, postoperative management, complications, functional outcomes, and follow-up.

Conclusion Management of recurrent LSCC after (C)RT is challenging and is based on the need to find a balance between oncologic and functional outcomes. Salvage $\mathrm{CO}_{2}$ TOLMS is a minimally invasive approach that can be applied to selected patients with strict and careful indications. Herein, a series of statements based on an ELS expert consensus aimed at guiding the main aspects of $\mathrm{CO}_{2}$ TOLMS for LSCC in the salvage setting is presented.
\end{abstract}

Keywords Carbon dioxide laser · Transoral laser microsurgery $\cdot$ Laryngeal cancer · Radiotherapy $\cdot$ Chemoradiation Salvage surgery

\section{Introduction}

Radiotherapy (RT) and chemoradiation (CRT) are wellestablished treatments for laryngeal squamous cell carcinoma (LSCC), with oncologic outcomes comparable with those obtained by surgery when appropriate patient selection has been accomplished. However, local recurrence after primary RT is not a rare event even in early-intermediate LSCC, ranging from 5 to 20 and from 25 to $30 \%$ in cT1 and cT2 lesions, respectively [1-5]. This poses a significant issue in patient management given the limited salvage treatment options after (C)RT. Laryngeal re-irradiation is rarely possible due to poor oncological and functional results [6-8], and

Alberto Paderno

albpaderno@gmail.com

Extended author information available on the last page of the article hence the need to opt for other treatments. Open-neck conservative options are hampered by RT-induced tissue modifications that frequently lead to complications and unpredictable recurrence patterns, possibly reducing the rates of laryngeal preservation and disease control. This prevented their widespread acceptance and application, while different groups confirmed their effectiveness in very selected cases even in the rescue setting [9-11]. As a consequence, in most instances, total laryngectomy is still considered the standard salvage treatment for patients previously treated with (C) RT experiencing LSCC recurrence. However, in such cases, ensuing complications and unavoidable functional sequelae (i.e., loss of physiologic phonation and swallowing impairment) almost invariably lead to a decreased quality of life.

For these reasons, carbon dioxide transoral laser microsurgery $\left(\mathrm{CO}_{2}\right.$ TOLMS $)$ definitively represents, when feasible, an extremely attractive option [12]. Several authors have confirmed the feasibility and oncological soundness 
of salvage $\mathrm{CO}_{2}$ TOLMS (Table 1) [13-28]. However, the suggested indications are highly variable and shared guidelines strongly needed. Therefore, the European Laryngological Society (ELS) selected an expert panel to discuss and reach consensus on key statements regarding preoperative management, treatment indications, and follow-up of LSCC after (C)RT. These statements represent the ensuing opinion coming from such a consensus and, in our view, could potentially guide the decision-making process in the salvage $\mathrm{CO}_{2}$ TOLMS scenario.

\section{Methods}

Members of the panel were selected from the ELS Committee on Laryngeal Cancer and Dysplasia (9 members, one of which declined participation) and other ELS members with proven experience in $\mathrm{CO}_{2}$ TOLMS until reaching the number of 15 . This number was agreed to ensure enough variation and, at the same time, allow an odds ratio for which a consensus was reached.

The initial statements were drafted by two authors (C.P. and A.P.) based on their personal experience and relevant literature. A modified Delphi survey was constructed for distribution throughout the group of authors (C.P., A.P., E.V.S., P.J.B, H.E.E., A.M., N.M., V.P., G.P., R.P., M.Q., M.R., V.V.P., I.V., and R.S.) to determine the consensus for each provisory recommendation [29].

\section{Modified Delphi process}

The modified Delphi process was utilized to determine which statements achieved full consensus. Through this method, each expert on the panel expressed his/her opinion for each proposed statement using a dedicated online survey. Responses for the modified Delphi survey were collected electronically. Results were analyzed using a Likert scale ranging from 1 to 9 , utilizing the following anchor points: 1 (strongly disagree), 3 (disagree), 5 (neutral), 7 (agree), and 9 (strongly agree). Statements were defined as achieving full consensus if there was a mean score of 7 or greater, and no outlier responses (scores lower than 5). Statements were considered achieving borderline consensus (thus needing modification and supplementary evaluation) when scores ranged between 6 and 7, or more than 7 with outlier responders. Statements were considered without consensus (and therefore deleted) when the score was less than 6 , regardless of the presence or not of outliers.

The process required a total of three rounds. In the first round, responses were double-blinded (to both responders and analyzers). In this phase, the statements without consensus were deleted, while those with borderline consensus were selected, modified to better specify the rationale of the items, and re-evaluated in a second round. The second round was single-blinded (only to responders) to allow subsequent feedback from those in disagreement (scores lower than 5). The third round consisted in personal communication among panel members to negotiate a final consensus.

\section{Results}

Response rates to the survey were $100 \%$ (15 of 15 panelists) for the first and second rounds, while only two members of the panel were involved in the third round.

\section{First round}

A total of 20 statements were drafted and submitted to all members. Among those, 13 reached a full consensus in this phase (mean score, 8.2; range 7.6-8.7). Conversely, one statement did not reach consensus (score, 5.9) and was therefore deleted, while 6 reached a borderline consensus due to insufficient score in 3 (mean score, 6.4; range 6.1-6.7), or outlier responses in 3. Statements reaching borderline consensus were subsequently modified and re-submitted in the second round.

\section{Second round}

A total of 6 statements, modified from the first round, were submitted to all members. Five reached full consensus (mean score, 7.9; range, 7.6-8.4), while one reached borderline consensus due to outlier responses by 2 panelists. The
Table 1 Oncologic outcomes of $\mathrm{CO}_{2}$ TOLMS in the salvage setting reported in the recent literature and including only series with 5-year results

\begin{tabular}{llllll}
\hline Authors & Year & No. of patients & T-categories & 5-year DSS & 5-year OS \\
\hline Quer et al. [14] & 2000 & 24 & rT1-rT2 & $100 \%$ & $76 \%$ \\
Steiner et al. [17] & 2004 & 34 & rT1-rT4 & $86 \%$ & $53 \%$ \\
Ansarin et al. [19] & 2007 & 37 & rTis-rT2 & - & $86 \%$ \\
Roedel et al. [22] & 2010 & 53 & rT1-rT3 & $68.6 \%$ & $53.3 \%$ \\
Han et al. [23] & 2012 & 18 & rT1-rT2 & $90 \%$ & $84.3 \%$ \\
Del Bon et al. [24] & 2012 & 35 & rT1-rT2 & $94 \%$ & $91 \%$ \\
Meulemans et al. [28] & 2018 & 33 & rT1-rT3 & $95.5 \%$ & $80.3 \%$ \\
\hline
\end{tabular}


statement not reaching full consensus was further discussed in the third round.

\section{Third round}

Consensus on the last statement was reached by direct discussion with the two experts in disagreement. After accurate evaluation and contextualization of the statement, consensus was reached without the need to modify the statement itself.

\section{Discussion and recommendations}

\section{Diagnostic work-up}

\section{Assessment of pre-(C)RT staging}

- The pre-(C)RT staging of the disease needs to be carefully assessed to determine the best salvage treatment. Recurrent lesions after RT originating from a primary tumor initially categorized as cT1 or cT2 without impairment of vocal fold mobility and/or anterior transcommissural extension can be considered for salvage $\mathrm{CO}_{2}$ TOLMS.

- Tumors initially categorized as cT2 with impaired vocal cord mobility, anterior transcommissural extension, cT3 for vocal cord/arytenoid fixation, or thyroid cartilage erosion, and failed after (C)RT should be considered suboptimal candidates for salvage $\mathrm{CO}_{2}$ TOLMS.

These considerations are related to the fact that tumor response to (C)RT does not induce a homogeneous concentric shrinkage of the lesion; instead, multiple resistant tumor foci may residue in otherwise healthy/cicatricial tissues [30]. The possibility of underestimation of the $\mathrm{T}$ category prior to (C)RT should be also taken into account. Consequently, safe resection margins can only be obtained by planning the excision in consideration of the initial tumor extent before (C)RT. This cannot be obtained if the primary tumor was not safely resectable by $\mathrm{CO}_{2}$ TOLMS in the first instance, as is especially the case for cT3 lesions with vocal cord/arytenoid fixation or thyroid cartilage erosion [31].

\section{Clinical and office-based assessment of rT}

- Preoperative evaluation should be performed by highdefinition videolaryngoscopy, possibly using biologic endoscopy techniques such as Narrow Band Imaging (NBI), Storz Professional Image Enhancement System (SPIES), I-Scan, or others.

- Preoperative prediction of laryngeal exposure can be of help in treatment planning to identify patients at a high risk of difficult laryngeal exposure.
Preoperative staging is a crucial step in planning salvage $\mathrm{CO}_{2}$ TOLMS. Underestimation of rT-category is a frequent issue due to diagnostic difficulties in the post-(C)RT setting [22]. In particular, RT-related inflammation and mucosal alterations may be a significant confounding factor leading to late identification of recurrent mucosal lesions. Endoscopy plays a pivotal role in diagnostic assessment of recurrence. Post-RT tissue modifications should be taken into account and distinguished from tumor relapse. In line with ELS recommendations for the follow-up of patients treated for LSCC [32], high-definition videolaryngoscopy, possibly integrated with video recording, storage of images, and use of bioendoscopy, is the most accurate clinical evaluation tool. This is especially true after (C)RT, thanks to the high accuracy of bioendoscopy in differentiating between neoplastic disease and post-RT inflammatory/cicatricial changes, even though mainly limited to the superficial aspect of the mucosal lining and needing a longer learning curve than that observed for naïve LSCC [33, 34]. Evaluation of vocal fold mobility is also essential to direct treatment indications. Impaired vocal fold mobility or arytenoid fixation can result from RTinduced damage of the vocal muscle or crico-arytenoid joint. However, it can also be a sign of deep tumor infiltration that needs to be discerned by specific radiologic evaluation [35].

Good laryngeal exposure is an essential consideration in previously irradiated patients and can be compromised because of reduced neck flexibility and tissue elasticity. The Laryngoscore and the mini-Laryngoscore are preoperative tools aimed at predicting laryngeal exposure by analyzing patient characteristics [36-39]. The Laryngoscore includes previous (C)RT as a negative factor for intraoperative exposure, and its influence should be considered together with other anatomical variables.

\section{Radiologic evaluation}

- Cross-sectional imaging is warranted in all patients with recurrent LSCC after (C)RT. Magnetic resonance (MR) should be preferred in view of its capability to better distinguish between inflammation, scar, and neoplastic tissue.

- Preoperative imaging by chest computed tomography (CT) scan, total body CT scan, or positron emission tomography (PET)/CT scan should be employed to exclude distant metastasis and synchronous tumors.

Submucosal tumor spread of recurrent LSCC is a frequent occurrence, thus hindering detection through endoscopic techniques. For these reasons, state-of-the-art radiologic imaging should be employed. MR offers higher contrast resolution than $\mathrm{CT}$, and is particularly helpful in distinguishing tumor infiltration from peritumoral inflammation and depicting cartilage invasion [40]. This is especially true 
using surface coils directly applied to the neck, designed to provide improved signal-to-noise ratios by limiting the spatial extent of the excitation and reception [35].

Finally, in the setting of persistent or recurrent LSCC after (C)RT, systemic imaging should be employed to rule out distant metastasis. PET/CT scan, carried out at least 12 weeks after treatment, demonstrated an adequate diagnostic potential in LSCC and head and neck cancer in general [41-43]. However, its effectiveness in detecting occult nodal metastases in recurrent LSCC remains debated [44]. Alternative imaging protocols (i.e., total body CT or chest CT scan) may be considered, especially in low-risk patients [45], in view of their minor cost and broader availability.

\section{Evaluation under general anesthesia}

- Tumor evaluation with high-definition rigid endoscopy (under white light and NBI/SPIES/I-Scan) and biopsy under general anesthesia should be considered in all patients with an indication to salvage $\mathrm{CO}_{2}$ TOLMS and indeterminate findings at the office-based evaluation.

- Evaluation of the real exposure and transoral full visibility of the entire tumor through the laryngoscope should ideally precede $\mathrm{CO}_{2}$ TOLMS of any rT to improve treatment planning and patient counseling.

Considering the potential difficulties and confounding factors related to office-based endoscopy after (C)RT, panendoscopy under general anesthesia should be performed with a very low threshold in all cases with indeterminate or suspicious findings at endoscopic and/or radiologic evaluation. Random biopsies should be avoided because of their limited diagnostic potential and risk of complications (e.g., softtissue edema, and chondritis with possible chondronecrosis). Conversely, biopsies should be precisely targeted (eventually following laser incision to reach submucosal tissues) to suspicious areas found using biologic endoscopy techniques.

Salvage $\mathrm{CO}_{2}$ TOLMS requires optimal laryngeal exposure given the need for a more extensive resection and difficult discrimination of tumor boundaries. In patients with a high risk of difficult laryngeal exposure at preoperative evaluation, assessment of exposure under general anesthesia is essential to direct the subsequent treatment approach. In all cases, optimal laryngeal exposure with a large-bore laryngoscope should be obtained, allowing adequate visualization up to the anterior commissure. Excellent laryngeal exposure is an absolute prerequisite for salvage $\mathrm{CO}_{2}$ TOLMS (even more than in the primary setting), since safe resection margins can usually be obtained only by more enlarged procedures (Type III-VI cordectomy) and accurate microscopic assessment. Patients presenting suboptimal exposure should be evaluated for alternative approaches regardless of their rT-category.

\section{Treatment indications}

\section{Glottic recurrence}

- Glottic LSCCs recurring after (C)RT and categorized as rcTis-T2 with normal vocal fold mobility can be considered for salvage $\mathrm{CO}_{2}$ TOLMS. However, pre-treatment staging and laryngeal exposure (see "Diagnostic workup") should be carefully taken into account.

- Glottic rT1b with anterior commissure involvement and rT2 with anterior transcommissural extension should be considered for $\mathrm{CO}_{2}$ TOLMS only in the presence of optimal laryngeal exposure.

Various authors described the oncologic outcomes of salvage $\mathrm{CO}_{2}$ TOLMS and proposed different indications in relation to complications and survival results [13-28]. Most series included rTis-T2 tumors; however, in some cases, indications were extended up to rT4a lesions [17, 22]. Treatment of T3-T4a glottic LSCC has been described in both primary and salvage $\mathrm{CO}_{2}$ TOLMS, but requires careful selection and significant surgical and technical expertise, being associated with unpredictable functional outcomes, notwithstanding the frequent need for complementary (C) $\mathrm{RT}$, which is clearly not repeatable in a salvage setting. Moreover, rT3-T4a lesions, as well as rT2 with impaired vocal fold mobility or anterior transcommissural extension, may have unexpected patterns of spread due to their deep infiltration, thus increasing the risk of local recurrence. In this setting, the results are strongly operator-dependent, and this factor prevents large-scale treatment adoption with homogeneous oncologic results. Furthermore, extensive removal of the perichondrium and resection of the cartilage can lead to impaired wound healing, chondronecrosis, and necrosis of soft tissues. These complications often result in severe functional impairment, impacting airway patency and swallowing, and may ultimately require total laryngectomy. The same holds true when considering recurrent LSCCs originating from advanced primary lesions (i.e., cT3-T4). Adequate treatment of these lesions including the pre- $(C)$ RT tumor margins would require a resection extending to the perichondrium or cartilage, thus incurring in the abovementioned issues.

As in the primary setting, management of tumors with anterior commissure involvement remains a debated issue. In this regard, oncologic results in the salvage setting are variable and their evaluation is severely impaired by the small sample size of the cohorts reported [15, 17, 19, 22]. In fact, some authors confirm anterior commissure involvement as a risk factor for recurrence, while others understate its risk potential. However, in view of the complex anatomical and technical management of this subsite [46], optimal laryngeal exposure should always be ensured before embarking 
into a salvage $\mathrm{CO}_{2}$ TOLMS addressing the region. This recommendation has also been confirmed by Steiner and coworkers, highlighting a lower laryngeal preservation rate in patients with anterior commissure involvement and suboptimal laryngeal exposure [17].

\section{Supraglottic recurrence}

- Supraglottic LSCCs categorized as rcTis-T2 can be effectively treated by salvage $\mathrm{CO} 2$ TOLMS.

As for glottic LSCC, various authors have described the technical feasibility of transoral supraglottic resection up to rT3 lesions $[13,14,21,25,26]$. However, the fibrocartilaginous laryngeal framework does not effectively stem bulky T3 and T4a tumors that invariably require resection of the hyoid bone, thyrohyoid membrane, thyroid cartilage, and soft tissues of the neck. Surgery in this setting becomes particularly challenging, hampering its widespread adoption with satisfying oncologic outcomes. The ensuing functional results are often suboptimal and worsened by frequent postoperative and long-term complications (e.g., chronic aspiration, pneumonia, chondronecrosis, and soft-tissue necrosis).

rcTis-T2 tumors may be more easily approached and treated by salvage $\mathrm{CO}_{2}$ TOLMS. However, patient selection plays a crucial role in treatment planning. Functional outcomes are invariably worse than in the primary setting, and patients should be able to tolerate chronic subclinical aspiration and a lengthy swallowing rehabilitation program. In particular, extensive resection of the aryepiglottic fold with arytenoidectomy should be avoided given the extremely high risk of postoperative acute and chronic aspiration [47].

\section{Regional recurrence}

- Concomitant local (rT $>1)$ and regional (rN $>1)$ recurrence after $(\mathrm{C}) \mathrm{RT}$ should be considered a suboptimal indication to salvage $\mathrm{CO}_{2}$ TOLMS.

Salvage $\mathrm{CO}_{2}$ TOLMS should not be considered as a first-line treatment for patients with extensive loco-regional recurrence of LSCC because of different factors. First of all, the presence of regional recurrence should be regarded as a sign of potential underestimation of the true primary and/ or recurrent tumor extension. Lateral neck metastasis may be related to undetected deep tumor extension and lymphovascular invasion.

Moreover, it is not possible to adequately address the lymphatic vessels connecting the tumor with pathologic lymph nodes (the so-called "T-N tract") through a purely transoral approach. In-transit metastatic cells may be left in the soft tissues between the endoscopic surgical field and the neck dissection specimen. Finally, patients treated by concomitant $\mathrm{CO}_{2}$ TOLMS and neck dissection have a significant risk of complications (such as fistula formation or subcutaneous emphysema) and postoperative laryngeal edema. In this view, the "cost-benefit ratio" of salvage $\mathrm{CO}_{2}$ TOLMS tends to increase dramatically in case of advanced lateral neck recurrence $(\mathrm{rN}>1)$, both in terms of oncologic results and complication rate.

\section{Postoperative management and complications}

- Prophylactic antibiotic therapy should be used to prevent postoperative infection/chondritis/chondronecrosis.

- Resumption of oral feeding is usually straightforward after glottic procedures; however, in supraglottic resections, there is often a need for nasogastric feeding tube for some days.

- Patients should be carefully monitored to detect early signs of perichondritis, chondritis, or chondronecrosis so that appropriate therapeutic measures can be instigated.

Salvage $\mathrm{CO}_{2}$ TOLMS can lead to reduced sensitivity and mobility of the larynx, resulting in an increased risk of aspiration during the early postoperative period. Patients undergoing supraglottic resections (particularly if reaching the aryepiglottic fold and/or the arytenoid) or extensive glottic procedures are at higher risk of aspiration and therefore prolonged use of a nasogastric feeding tube [47].

Perichondral damage following $\mathrm{CO}_{2}$ TOLMS may allow bacteria to gain access to the cartilage with possible infection. This may lead to perichondritis, an inflammatory process of the perichondrium that precedes chondritis and chondronecrosis. In hypovascularized and irradiated tissues, infection initiates a vicious cycle leading to tissue damage and hypoxia, compromised vascularity, and further progression of the infective/inflammatory process [48, 49]. Consequently, chondronecrosis is significantly more likely in patients who received high-dose RT combined with exposure or disruption of the perichondrium. For this reason, prophylactic antibiotic therapy may be helpful in patients receiving resections exposing the perichondrium, while no data are yet available on the utility of this aid.

Once developed, laryngeal chondronecrosis is often irreversible. Total laryngectomy is frequently required because of life-threatening bleeding, laryngeal framework collapse, and ensuing airway obstruction. Chondronecrosis should be monitored and evaluated according to the grading system proposed by Chandler et al. [50]. Grades I and II are common post-RT changes and typically respond favorably to conservative treatments (i.e., humidification, voice restraint, discontinuation of smoking, and antibiotics), while Grade III and IV reactions are more severe and have less favorable outcomes. Perichondritis, chondritis, and chondronecrosis may respond well to hyperbaric oxygen therapy, even though 
persistent tumor should be absolutely ruled out before considering this treatment to avoid hyperbaric oxygen-induced tumor acceleration [51, 52]. However, Grades III and IV are significantly less likely to be successfully treated by a conservative approach.

Complications of salvage $\mathrm{CO}_{2}$ TOLMS reported in the recent literature are summarized in Table 2.

\section{Functional outcomes}

- Tracheotomy and gastrostomy tube placement are infrequently, and usually only temporarily, needed in the salvage $\mathrm{CO}_{2}$ TOLMS setting.

- Vocal outcomes after salvage $\mathrm{CO}_{2}$ TOLMS are usually inferior to those obtained in the primary setting. Moderate or severe dysphonia should be expected.

- A slower and more gradual resumption of swallowing should be expected in supraglottic resections compared with purely glottic procedures.

The length of feeding tube dependency and chronic aspiration rate are significantly higher in salvage $\mathrm{CO}_{2}$ TOLMS [53]. Nevertheless, according to a recent systematic review, postoperative tracheotomy and gastrostomy in patients treated by $\mathrm{CO}_{2}$ TOLMS were needed only in $2.3 \%$ and $6.6 \%$ of patients, respectively [54]. Furthermore, several studies have reported higher gastrostomy tube dependency rates in supraglottic recurrences compared to glottic ones [25, 26]. However, the number of patients involved was not sufficient for precise and reliable comparisons.

Objective data assessing vocal outcomes in salvage $\mathrm{CO}_{2}$ TOLMS are scarce and heterogeneous. In general, the results seem to be inferior to those in the primary setting, while a small study providing a direct comparison did not find significant differences [24]. In a study by Puxeddu and coworkers, postoperative perceptual voice evaluation (grade, roughness, breathiness, asthenia, and strain score) showed mild, moderate, and severe dysphonia in $12.5 \%, 25 \%$, and $37.5 \%$ of patients, respectively. Furthermore, videolaryngostroboscopy showed incomplete glottic closure in 56\% of patients [18].

\section{Follow-up}

- Follow-up policy should strictly observe guidelines provided by the ELS, with particular attention to the indications after $(\mathrm{C}) \mathrm{RT}$.

Follow-up has a critical role in the overall management of patients treated by salvage $\mathrm{CO}_{2}$ TOLMS. In fact, recurrences are more frequent and less easily detected than in the primary setting. All measures described in the "Diagnostic work-up" section should be undertaken when evaluating a newly developed lesion or functional alteration. In adjunction, the development of treatment-related complications should also be monitored in the medium and long terms.

The ELS has proposed a series of recommendations for the follow-up of patients treated for LSCC to provide an up-to-date, evidence-based protocol that is meaningful and applicable to all European health care systems [32]. These recommendations represent an optimal framework from which to structure patient follow-up after salvage $\mathrm{CO}_{2}$ TOLMS.

\section{Conclusions}

Management of recurrent LSCC after (C)RT is particularly challenging and requires careful evaluation to select the optimal therapeutic option according to the characteristics of the patient and tumor. In this setting, $\mathrm{CO}_{2}$ TOLMS has the crucial role to allow a conservative and minimally invasive

Table 2 Complications reported in the recent literature

\begin{tabular}{|c|c|c|c|}
\hline Authors & Year & No. of patients & Reported complications \\
\hline Quer et al. [14] & 2000 & 24 & Laryngeal stenosis $(N=2)$ \\
\hline De Gier et al. [15] & 2001 & 44 & Chondritis $(N=2)$ \\
\hline Steiner et al. [17] & 2004 & 34 & $\begin{array}{l}\text { Synechiae }(N=3) \text {, aspiration pneumonia }(N=1) \text {, chondronecrosis }(N=1) \text {, laryngeal stenosis } \\
(N=1)\end{array}$ \\
\hline Puxeddu et al. [18] & 2004 & 16 & None \\
\hline Ansarin et al. [19] & 2007 & 37 & Laryngeal stenosis $(N=4)$ \\
\hline Roedel et al. [22] & 2010 & 53 & $\begin{array}{l}\text { Laryngeal edema }(N=5) \text {, synechiae }(N=4) \text {, laryngeal stenosis }(N=3) \text {, postoperative bleeding } \\
(N=2) \text {, chondronecrosis }(N=1)\end{array}$ \\
\hline Han et al. [23] & 2012 & 18 & $\begin{array}{l}\text { Excessive granulation tissue }(N=3) \text {, temporary lingual numbness }(N=2) \text {, temporary hypoglossal } \\
\text { palsy }(N=1)\end{array}$ \\
\hline Del Bon et al. [24] & 2012 & 35 & Chondronecrosis $(N=2)$, chondritis $(N=1)$, postoperative bleeding $(N=1)$ \\
\hline Abouyared et al. [26] & 2014 & 52 & Prolonged postoperative pain $(N=13)$, chondronecrosis $(N=12)$ \\
\hline Fink et al. [27] & 2016 & 42 & Laryngeal edema $(N=1)$ \\
\hline
\end{tabular}


approach even after failure of primary non-surgical therapy. However, when $\mathrm{CO}_{2}$ TOLMS is the preferred salvage treatment, patient selection is essential to achieve satisfying oncologic and functional results. Therefore, diagnostic workup, treatment indications, and postoperative management should be optimized according to the available evidence. In consideration of the scarcity of data in the current literature, the statements collected in this manuscript and reflecting the expert opinion of a panel of European laryngologists may significantly help this process and favor standardization in management of patients treated by salvage $\mathrm{CO}_{2}$ TOLMS.

Acknowledgements The data that support the findings of this study are available from the corresponding author upon reasonable request.

Funding Open access funding provided by Università degli Studi di Brescia within the CRUI-CARE Agreement.

\section{Declarations}

Conflict of interest The authors declare no conflict of interest.

Open Access This article is licensed under a Creative Commons Attribution 4.0 International License, which permits use, sharing, adaptation, distribution and reproduction in any medium or format, as long as you give appropriate credit to the original author(s) and the source, provide a link to the Creative Commons licence, and indicate if changes were made. The images or other third party material in this article are included in the article's Creative Commons licence, unless indicated otherwise in a credit line to the material. If material is not included in the article's Creative Commons licence and your intended use is not permitted by statutory regulation or exceeds the permitted use, you will need to obtain permission directly from the copyright holder. To view a copy of this licence, visit http://creativecommons.org/licenses/by/4.0/.

\section{References}

1. Sewnaik A, Meeuwis CA, van der Kwast TH, Kerrebijn JD (2005) Partial laryngectomy for recurrent glottic carcinoma after radiotherapy. Head Neck 27:101-107

2. Mendenhall WM, Parsons JT, Million RR, Fletcher GH (1988) T1-T2 squamous cell carcinoma of the glottic larynx treated with radiation therapy: relationship of dose-fractionation factors to local control and complications. Int J Radiat Oncol Biol Phys $15: 1267-1273$

3. Moose BD, Greven KM (1997) Definitive radiation management for carcinoma of the glottic larynx. Otolaryngol Clin North Am 30:131-143

4. Raitiola H, Wigren T, Pukander J (2000) Radiotherapy outcome and prognostic factors in early glottic carcinoma. Auris Nasus Larynx 27:153-159

5. Nur DA, Oguz C, Kemal ET et al (2005) Prognostic factors in early glottic carcinoma implications for treatment. Tumori 91:182-187

6. Langendijk JA, Kasperts N, Leemans CR, Doornaert P, Slotman BJ (2006) A phase II study of primary reirradiation in squamous cell carcinoma of head and neck. Radiother Oncol 78:306-312
7. Strojan P, Corry J, Eisbruch A et al (2015) Recurrent and second primary squamous cell carcinoma of the head and neck: when and how to reirradiate. Head Neck 37:134-150

8. Dionisi F, Fiorica F, D'Angelo E et al (2019) Organs at risk's tolerance and dose limits for head and neck cancer re-irradiation: a literature review. Oral Oncol 98:35-47

9. Pellini R, Pichi B, Ruscito P, Ceroni AR et al (2008) Supracricoid partial laryngectomies after radiation failure: a multi-institutional series. Head Neck 30:372-379

10. De Virgilio A, Pellini R, Mercante G et al (2018) Supracricoid partial laryngectomy for radiorecurrent laryngeal cancer: a systematic review of the literature and meta-analysis. Eur Arch Otorhinolaryngol 275:1671-1680

11. Bertolin A, Lionello M, Ghizzo M et al (2020) Salvage open partial horizontal laryngectomy after failed radiotherapy: a multicentric study. Laryngoscope 130:431-436

12. Remacle M, Arens C, Eldin MB et al (2017) Laser-assisted surgery of the upper aero-digestive tract: a clarification of nomenclature. A consensus statement of the European Laryngological Society. Eur Arch Otorhinolaryngol 274:3723-3727

13. Outzen KE, Illum P (1995) CO2-laser therapy for carcinoma of the larynx. J Laryngol Otol 109:111-113

14. Quer M, León X, Orús C, Venegas P, López M, Burgués J (2000) Endoscopic laser surgery in the treatment of radiation failure of early laryngeal carcinoma. Head Neck 22:520-523

15. de Gier HH, Knegt PP, de Boer MF, Meeuwis CA, van der Velden LA, Kerrebijn JD (2001) CO2-laser treatment of recurrent glottic carcinoma. Head Neck 23:177-180

16. Pukander J, Kerälä J, Mäkitie A, Hyrynkangas K, Virtaniemi J, Grenman R (2001) Endoscopic laser surgery for laryngeal cancer. Eur Arch Otorhinolaryngol 258:236-239

17. Steiner W, Vogt P, Ambrosch P, Kron M (2004) Transoral carbon dioxide laser microsurgery for recurrent glottic carcinoma after radiotherapy. Head Neck 26:477-484

18. Puxeddu R, Piazza C, Mensi MC, Ledda GP, Argiolas F, Peretti G (2004) Carbon dioxide laser salvage surgery after radiotherapy failure in T1 and T2 glottic carcinoma. Otolaryngol Head Neck Surg 130:84-88

19. Ansarin M, Planicka M, Rotundo S et al (2007) Endoscopic carbon dioxide laser surgery for glottic cancer recurrence after radiotherapy: oncological results. Arch Otolaryngol Head Neck Surg 133:1193-1197

20. Piazza C, Peretti G, Cattaneo A, Garrubba F, De Zinis LOR, Nicolai P (2007) Salvage surgery after radiotherapy for laryngeal cancer: from endoscopic resections to open-neck partial and total laryngectomies. Arch Otolaryngol Head Neck Surg 133:1037-1043

21. Grant DG, Salassa JR, Hinni ML, Pearson BW, Hayden RE, Perry WC (2008) Transoral laser microsurgery for recurrent laryngeal and pharyngeal cancer. Otolaryngol Head Neck Surg 138:606-613

22. Roedel RM, Matthias C, Wolff HA, Schindler P, Aydin T, Christiansen $H$ (2010) Transoral laser microsurgery for recurrence after primary radiotherapy of early glottic cancer. Auris Nasus Larynx 37:474-481

23. Han YJ, Lee HS, Kim SW et al (2012) Transoral laser microsurgery of recurrent early glottic cancer after radiation therapy: clinical feasibility and limitations. Ann Otol Rhinol Laryngol 121:375-382

24. Del Bon F, Piazza C, Mangili S, De Zinis LR, Nicolai P, Peretti G (2012) Transoral laser surgery for recurrent glottic cancer after radiotherapy: oncologic and functional outcomes. Acta Otorhinolaryngol Ital 32:229

25. Reynolds L, Rigby M, Trites J, Hart R, Taylor S (2013) Outcomes of transoral laser microsurgery for recurrent head and neck cancer. J Laryngol Otol 127:982-986 
26. Abouyared M, Ojo R, Fundakowski C, Lo K, Sargi Z (2014) Transoral laser microsurgery in previously irradiated patients with laryngeal cancer. Am J Otolaryngol 35:279-285

27. Fink DS, Sibley H, Kunduk M et al (2016) Functional outcomes after salvage transoral laser microsurgery for laryngeal squamous cell carcinoma. Otolaryngol Head Neck Surg 155:606-611

28. Meulemans J, Bijnens J, Delaere P, Vander Poorten V (2018) Upfront and salvage transoral laser microsurgery for early glottic squamous cell carcinoma: a single centre retrospective case series. Front Oncol 8:186

29. Dalkey N, Helmer O (1963) An experimental application of the Delphi method to the use of experts. Manag Sci 9:458-467

30. Zbaren P, Nuyens M, Curschmann J, Stauffer E (2007) Histologic characteristics and tumor spread of recurrent glottic carcinoma: analysis on whole-organ sections and comparison with tumor spread of primary glottic carcinomas. Head Neck 29:26-32

31. Vilaseca I, Nogues-Sabate A, Aviles-Jurado FX et al (2019) Factors of local recurrence and organ preservation with transoral laser microsurgery in laryngeal carcinomas; CHAID decision-tree analysis. Head Neck 41:756-764

32. Simo R, Bradley P, Chevalier D et al (2014) European Laryngological Society: ELS recommendations for the follow-up of patients treated for laryngeal cancer. Eur Arch Otorhinolaryngol 271:2469-2479

33. Piazza C, Cocco D, De Benedetto L, Del Bon F, Nicolai P, Peretti G (2010) Narrow band imaging and high definition television in the assessment of laryngeal cancer: a prospective study on 279 patients. Eur Arch Otorhinolaryngol 267:409-414

34. Piazza C, Del Bon F, Peretti G, Nicolai P (2012) Narrow band imaging in endoscopic evaluation of the larynx. Curr Opin Otolaryngol Head Neck Surg 20:472-476

35. Ravanelli M, Paderno A, Del Bon F et al (2019) Prediction of posterior paraglottic space and cricoarytenoid unit involvement in endoscopically $\mathrm{T} 3$ glottic cancer with arytenoid fixation by magnetic resonance with surface coils. Cancers (Basel) 11:67

36. Arjun AP, Dutta A (2019) A study of application of preoperative clinical predictors of difficult laryngeal exposure for microlaryngoscopy: the Laryngoscore in the Indian population. Indian J Otolaryngol Head Neck Surg 71:480-485

37. Incandela F, Paderno A, Missale F et al (2019) Glottic exposure for transoral laser microsurgery: Proposal of a mini-version of the laryngoscore. Laryngoscope 129:1617-1622

38. Piazza C, Mangili S, Bon FD et al (2014) Preoperative clinical predictors of difficult laryngeal exposure for microlaryngoscopy: the Laryngoscore. Laryngoscope 124:2561-2567

39. Tirelli G, Gatto A, Fortunati A, Marzolino R, Giudici F, Boscolo Nata F (2019) Predicting laryngeal exposure in microlaryngoscopy: external validation of the laryngoscore. Laryngoscope 129:1438-1443

40. Maroldi R, Ravanelli M, Farina D (2014) Magnetic resonance for laryngeal cancer. Curr Opin Otolaryngol Head Neck Surg 22:131-139

41. Tatar G, Cermik TF, Karagoz Y et al (2018) The value of wholebody contrast-enhanced 18F-FDG PET/CT imaging in the diagnosis and staging of patients with laryngeal carcinoma. Nucl Med Commun 39:334-342

42. Mehanna H, Wong WL, McConkey CC et al (2016) PET-CT surveillance versus neck dissection in advanced head and neck cancer. N Engl J Med 374:1444-1454

43. Seebauer CT, Hackenberg B, Grosse J et al (2021) Routine restaging after primary non-surgical treatment of laryngeal squamous cell carcinoma-a review. Strahlenther Onkol 197:167-176

44. Rosko A, Birkeland A, Shuman A et al (2017) Positron emission tomography-CT prediction of occult nodal metastasis in recurrent laryngeal cancer. Head Neck 39:980-987

45. Spector JG, Sessions DG, Haughey BH et al (2001) Delayed regional metastases, distant metastases, and second primary malignancies in squamous cell carcinomas of the larynx and hypopharynx. Laryngoscope 111:1079-1087

46. Piazza C, Filauro M, Paderno A et al (2018) Three-dimensional map of isoprognostic zones in glottic cancer treated by transoral laser microsurgery as a unimodal treatment strategy. Front Oncol 8:175

47. Piazza C, Barbieri D, Del Bon F et al (2016) Functional outcomes after different types of transoral supraglottic laryngectomy. Laryngoscope 126:1131-1135

48. Kashima HK, Holliday MJ, Hyams VJ (1977) Laryngeal chondronecrosis: clinical variations and comments on recognition and management. Trans Sect Otolaryngol Am Acad Ophthalmol Otolaryngol 84:ORL878-ORL881

49. Pang L, Jeannon JP, Simo R (2011) Minimizing complications in salvage head and neck oncological surgery following radiotherapy and chemo-radiotherapy. Curr Opin Otolaryngol Head Neck Surg 19:125-131

50. Chandler JR (1979) Radiation fibrosis and necrosis of the larynx. Ann Otol Rhinol Laryngol 88:509-514

51. Ferguson BJ, Hudson WR, Farmer JC Jr (1987) Hyperbaric oxygen therapy for laryngeal radionecrosis. Ann Otol Rhinol Laryngol 96:1-6

52. Filntisis GA, Moon RE, Kraft KL, Farmer JC, Scher RL, Piantadosi CA (2000) Laryngeal radionecrosis and hyperbaric oxygen therapy: report of 18 cases and review of the literature. Ann Otol Rhinol Laryngol 109:554-562

53. Hutcheson KA, Jantharapattana K, Barringer DA, Lewin JS, Holsinger FC (2012) Functional and oncological outcomes of primary versus salvage transoral laser microsurgery for supraglottic carcinoma. Ann Otol Rhinol Laryngol 121:664-670

54. Meulemans J, Delaere P, Nuyts S, Clement PM, Hermans R, Vander Poorten V (2017) Salvage transoral laser microsurgery for radiorecurrent laryngeal cancer: Indications, limits, and outcomes. Curr Otorhinolaryngol Rep 5:83-91

Publisher's Note Springer Nature remains neutral with regard to jurisdictional claims in published maps and institutional affiliations.

\title{
Authors and Affiliations
}

\author{
Cesare Piazza ${ }^{1,2}$ - Alberto Paderno ${ }^{1,2}$ (1) $\cdot$ Elisabeth V. Sjogren ${ }^{3} \cdot$ Patrick J. Bradley $^{4}$ Hans E. Eckel ${ }^{5} \cdot$ Antti Mäkitie $^{6}$. \\ Nayla Matar ${ }^{7} \cdot$ Vinidh Paleri $^{8}$. Giorgio Peretti ${ }^{9} \cdot$ Roberto Puxeddu $^{10} \cdot$ Miquel Quer $^{11} \cdot$ Marc Remacle $^{12}$. \\ Vincent Vander Poorten ${ }^{13,14} \cdot$ Isabel Vilaseca ${ }^{15} \cdot$ Ricard Simo $^{16}$
}


1 Department of Otorhinolaryngology-Head and Neck Surgery, ASST — Spedali Civili di Brescia, University of Brescia, Piazza Spedali Civili 1, 25123 Brescia, Italy

2 Department of Medical, Surgical and Radiological Sciences and Public Health, University of Brescia, Brescia, Italy

3 Department of Otolaryngology—Head and Neck Surgery, Leiden University Medical Centre, Leiden, The Netherlands

4 Department of ORL-HNS, Queens Medical Centre Campus, Nottingham University Hospitals, Derby Road, Nottingham, UK

5 Department of Oto-Rhino-Laryngology-Head and Neck Surgery, Klagenfurt General Hospital, Klagenfurt, Austria

6 Department of Otorhinolaryngology-Head and Neck Surgery, University of Helsinki and HUS Helsinki University Hospital, Helsinki, Finland

7 Department of Otolaryngology-Head and Neck Surgery, Saint Joseph University, Hotel Dieu de France Hospital, Beirut, Lebanon

8 Head and Neck Unit, The Royal Marsden NHS Foundation Trust, London, UK
9 Department of Otorhinolaryngology-Head and Neck Surgery, IRCCS Ospedale Policlinico San Martino, University of Genoa, Genoa, Italy

10 Unit of Otorhinolaryngology-Head and Neck Surgery, Azenda Ospedaliero Universitaria, Presidio Ospedaliero Duilio Casula, Cagliari, Italy

11 Department of Otolaryngology-Head and Neck Surgery, University Hospital de la Santa Creu i Sant Pau, Universitat Autonòma de Barcelona, Barcelona, Spain

12 Department of Otolaryngology, Centre Hospitalier du Luxembourg, Luxembourg, Luxembourg

13 Department of Otorhinolaryngology-Head and Neck Surgery, Leuven Cancer Institute, University Hospitals Leuven, Leuven, Belgium

14 Department of Oncology, Section Head and Neck Oncology, KU Leuven, Leuven, Belgium

15 Otorhinolaryngology Service, Hospital Clinic de Barcelona, Universitat de Barcelona, Barcelona, Spain

16 Department of Otorhinolaryngology-Head and Neck Surgery, Head, Neck and Thyroid Cancer Unit, Guy's and St Thomas Hospital NHS Foundation Trust, London, UK 